\title{
Use of Levetiracetam to Treat Tics in Children and Adolescents With Tourette Syndrome
}

\author{
Yasser Awaad, MD, MSc, ${ }^{*}$ Anne Marie Michon, MSN, RN, and Sarah Minarik, BSN, RN \\ Department of Pediatrics, Oakwood Healthcare System, University of Michigan Medical School, Dearborn, Michigan, USA
}

\begin{abstract}
Some drugs currently used to treat tics in pediatric patients have drawbacks, including the risk of side effects. New therapeutic options with better safety profiles are needed. Levetiracetam is an antiepileptic drug with atypical mechanisms of action that might be beneficial for this indication. We evaluated the effects of levetiracetam on motor and vocal tics, behavior, and school performance in children and adolescents with tics and Tourette syndrome (TS). Sixty patients, $\leq 18$ years of age, with tics and TS were enrolled in this prospective, open-label study. The initial starting dose of levetiracetam was $250 \mathrm{mg} / \mathrm{day}$. The dosage was titrated over 3 weeks to 1,000 to 2,000 mg/day. Clinical outcomes were assessed with the Clin-
\end{abstract}

ical Global Impression Scale, Yale Global Tic Severity Scale, and Revised Conners' Parent Rating Scale. Behavior and school performance were also recorded. All 60 patients showed improvements based on all of the scales used, and 43 patients improved with regard to behavior and school performance. Levetiracetam was generally well tolerated. Three patients discontinued treatment because of exaggeration of preexisting behavioral problems. Levetiracetam may be useful in treating tics in children and adolescents. Given its established safety profile, levetiracetam is a candidate for evaluation in a wellcontrolled trial. (C) 2005 Movement Disorder Society

Key words: tics; Tourette syndrome; levetiracetam
Tics are a common movement disorder in children and adolescents. As many as $10 \%$ of boys may experience tics at some point during childhood; tics are less common in girls. ${ }^{1-3}$ Both motor tics (e.g., involving the facial muscles, neck, and upper limbs) and vocal tics (e.g., throat clearing, grunting, coughing, or cursing) occur in patients with Tourette syndrome (TS). In addition, patients with TS often have comorbidities such as obsessive-compulsive disorder and attention deficit hyperactivity disorder (ADHD). ${ }^{4}$

Although tics may spontaneously improve or resolve over time, drug therapy should be considered for patients whose symptoms interfere with their daily life. A major goal of therapy for tics is to improve the patient's quality of life by minimizing the potential for social embarrassment. Drugs used to treat TS include the neuroleptic agents haloperidol, pimozide, and fluphenazine..$^{5-7}$ How-

*Correspondence to: Dr. Yasser Awaad, University of Michigan Medical School, 21031 Michigan Avenue, Dearborn, MI 48124.

E-mail: yasser.awaad@oakwood.org

Received 12 April 2004; Revised 11 August 2004; Accepted 21 September 2004

Published online 9 February 2005 in Wiley InterScience (www. interscience.wiley.com). DOI: 10.1002/mds.20385 ever, the use of these drugs is associated with troubling side effects. In one study, for example, only $12.5 \%$ (3 of 24) of patients with tics were able to continue their haloperidol without interruption. ${ }^{8}$ Because of issues with neuroleptic drugs, other agents that are less effective but believed to be better tolerated are being evaluated for the treatment of tics. Despite the evaluation of several drugs, no single drug has emerged as an ideal therapy. Thus, there continues to be a need for effective and safe agents to treat tics.

Based on the proposed role of $\gamma$-aminobutyric acid (GABA) in the dysfunction of the basal ganglia in patients with $\mathrm{TS}, 7,9$ GABAergic drugs may be a viable therapeutic option to improve tics. GABAergic drugs such as clonazepam and baclofen, for example, have been evaluated. ${ }^{7,10}$ Levetiracetam is an antiepileptic drug that may have atypical GABAergic effects. ${ }^{11}$ Levetiracetam inhibits the ability of zinc and beta carbolines to interrupt chloride influx - an effect that enhances chloride ion influx at the GABA type $\mathrm{A}$ (GABA-A) receptor complex. ${ }^{12}$ These mechanisms of action could produce a beneficial effect in patients with TS. In addition, the safety profile of levetiracetam is well established, and there are some studies of the use of levetiracetam in 
children. ${ }^{13-19}$ Thus, levetiracetam may be a better-tolerated alternative to currently used therapies.

The effect of levetiracetam on motor and vocal tics was studied in an open-label manner in a population of children and adolescents with tics and TS. In addition, the effects of levetiracetam therapy on measures of behavior and school performance were recorded.

\section{PATIENTS AND METHODS}

\section{Patients}

Sixty patients ( $\leq 18$ years of age) with tics and TS who were treated at the Oakwood Healthcare System in Michigan were enrolled in a prospective, open-label study. All patients met the DSM-IV criteria for tics and TS. ${ }^{20}$ All patients were newly diagnosed and, thus, had not been previously treated for TS. The period between diagnosis and study entry was 1 to 2 months. Appropriate informed consent was obtained for each patient.

\section{Dosing and Administration}

The initial dose of levetiracetam was $250 \mathrm{mg} /$ day (p.o.). The dosage was titrated over a 3-week period up to 1,000 to $2,000 \mathrm{mg} / \mathrm{day}$, based on response as well as tolerability. The dosage was usually increased by $250 \mathrm{mg}$ every 5 to 7 days. Age and body weight were considerations in dosing based on published information regarding the use of levetiracetam in children. ${ }^{13,16}$ At the time of this analysis, patients were treated with levetiracetam for 1 year. No concomitant medications were used to treat tics.

\section{Measures of Clinical Efficacy}

Clinical outcomes were assessed with the Clinical Global Impression Scale (CGI), Yale Global Tic Severity Scale (YGTSS), and Revised Conners' Behavior Scales, including the Revised Conners' Teacher Rating Scale (CTRS-R). Evaluations were completed at baseline and 3,6 , and 12 months after initiation of therapy.

The CGI is a seven-point scale based on all available information concerning the adverse impact of tic behavior on the patient's life. The YGTSS consists of the separate rating of severity for motor and vocal tics along five discriminate dimensions. A scale of 0 to 5 is used for each dimension, including number, frequency, intensity, complexity, and interference. The summation of these scores (0 to 50) produces a Total Tic Score (TTS). The Tic Impairment Score (TIS), based on the impact of the tic disorder on areas such as self-esteem, family life, social acceptance, and school performance, is added to the TTS to obtain the total YGTSS score.

The Revised Conners' Behavior Scales assess problem behaviors reported by teachers, parents, other caregivers,

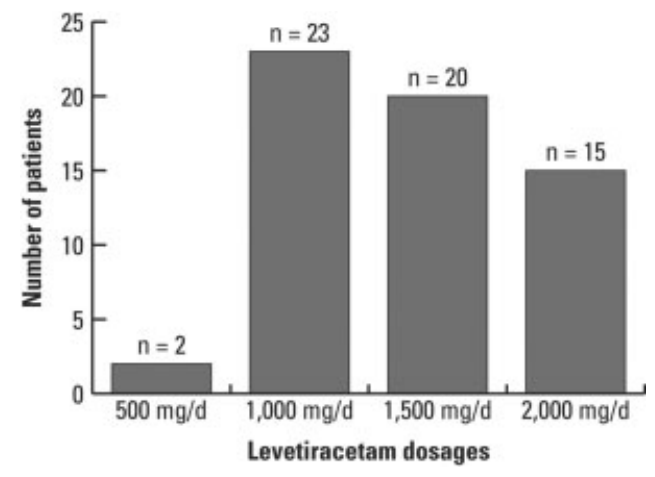

FIG. 1. Dosing of levetiracetam.

and adolescent patients. The Revised Conners' Parent Rating Scale (CPRS-R) and the CTRS-R include scales pertaining to ADHD behaviors, inattention, and hyperactivity/impulsivity. The 27-item CPRS-R (known as the short form or CPRS-R:S) includes five scales, and the 28-item CTRS-R (known as the short form or CTRS$\mathrm{R}: \mathrm{S})$ includes four scales. The Conner-Wells' Adolescent Self-Report Scale-Short version was used to assess self-reports of problem behaviors.

Other assessments of behavior and school performance were completed by reviewing report cards, progress reports, and improvements in grade point average. Parent interviews were also used to evaluate the behavior and school performance of the patients.

\section{Statistical Analyses}

Changes from baseline to 1 year after treatment on scales of clinical outcomes were analyzed using SPSS $v$. 10.0 software (SPSS, Chicago, IL). Differences were assessed using the two-tailed paired $t$ test.

\section{RESULTS}

\section{Demographic Characteristics}

The mean age of patients enrolled in this trial was 12 years, with a range of 6 to 18 years of age. The patient population was predominantly male, with 45 boys (75\%) and 15 girls (25\%).

\section{Dosing of Levetiracetam}

Most patients $(\mathrm{n}=23)$ were treated with $1,000 \mathrm{mg} /$ day levetiracetam (Fig. 1). There were 2 patients who were treated with $500 \mathrm{mg} /$ day, 20 with $1,500 \mathrm{mg} /$ day, and 15 with $2,000 \mathrm{mg} /$ day.

\section{Clinical Improvement}

All 60 patients showed significant clinical improvements in their vocal and motor tics as determined by the 


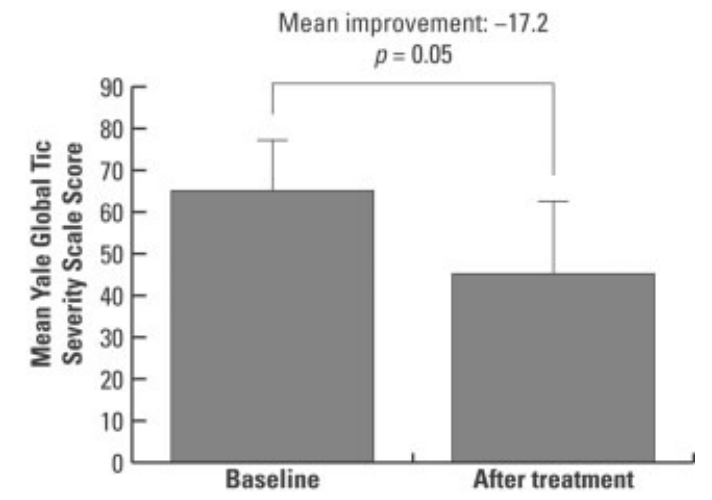

FIG. 2. Mean Yale Global Tic Severity Scale score at baseline and after treatment with levetiracetam.

YGTSS, TIS, TTS, and CGI scales $(P<0.05$; Figs. 2-5).

A total of 43 patients showed improvement in their behavior and school performance as determined by the CPRS-R $(P<0.04)$ and the Conner-Wells' Adolescent Self-Report Scale-Short version $(P<0.05)$. In addition, grade point averages improved from $1.5 \pm 1.0$ to $2.5 \pm$ 0.5 .

\section{Safety and Tolerability of Levetiracetam Therapy}

With regard to mean, aggressive, and impulsive behavior, 3 patients had baseline behavior or ADHD symptoms, or both. These 3 patients withdrew from the study because of exaggeration of their behavior problems.

Somnolence was experienced by 4 patients. In 3 patients, this somnolence dissipated over time. In the fourth patient, the dose of levetiracetam was reduced and titrated more slowly. There were 2 patients who reported asthenia and 5 reported headache.

\section{DISCUSSION}

Tics and TS can impair the quality of life of children and adolescents because of social embarrassment and

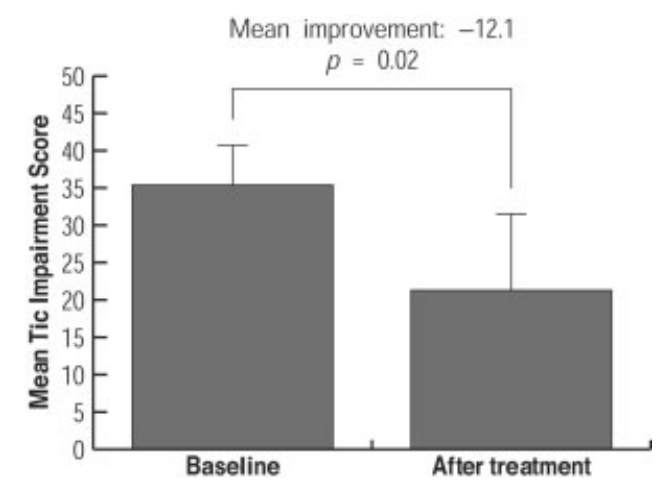

FIG. 3. Mean Tic Impairment Score at baseline and after treatment with levetiracetam.

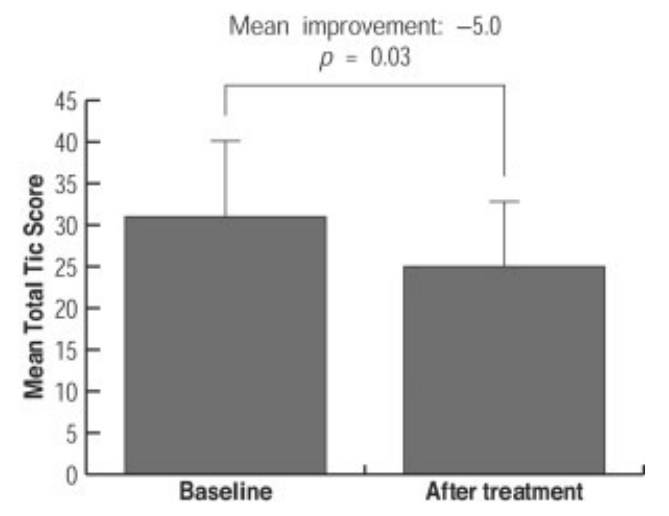

FIG. 4. Mean Total Tic Score at baseline and after treatment with levetiracetam.

symptoms that interfere with their daily functioning. Thus, it is important to address these conditions, given that they often occur during critical periods of learning and development.

Several drugs have been evaluated for the treatment of TS. Treatment with haloperidol results in improvement in an estimated $70 \%$ to $80 \%$ of patients. ${ }^{6}$ However, haloperidol is associated with potentially serious side effects such as tardive dyskinesia. More recently introduced atypical neuroleptics, including risperidone, quetiapine, olanzapine, and clozapine, may have fewer or different side effects but are still less than optimal. Children may be more vulnerable to adverse effects of neuroleptic agents, including sedation, weight gain, and extrapyramidal symptoms than adults. ${ }^{21}$

The $\alpha-2$ adrenergic agents clonidine and guanfacine are also used to treat tic disorders. ${ }^{4,10}$ Although these drugs may not be as effective as neuroleptic agents, their side-effect profiles are preferable to those of neuroleptic drugs. Several antidepressants, ${ }^{6}$ and other agents, such as the opioid antagonist naloxone, have been used to treat tics. None of these drugs has emerged as an ideal therapy for TS, and new treatment options are being evaluated.

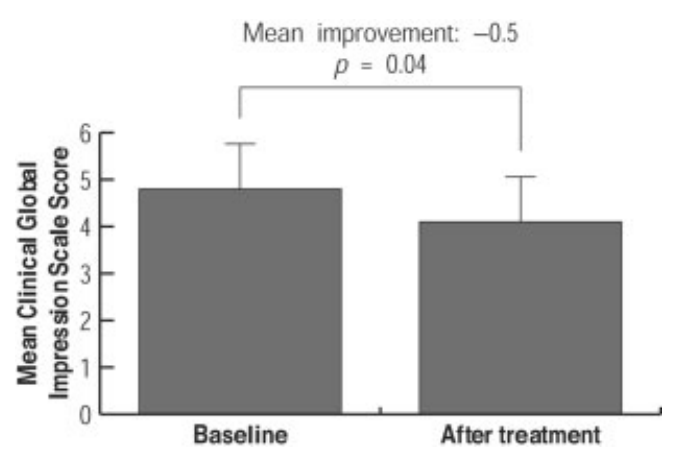

FIG. 5. Mean Clinical Global Impression Scale score at baseline and after treatment with levetiracetam. 
The pathophysiology of TS is not fully elucidated, but existing evidence suggests that disorders of the basal ganglia play a role in this syndrome. ${ }^{7,9}$ Data showing that the GABAergic areas of the basal ganglia are defective in TS provide a rationale for the evaluation of drugs with specific GABAergic effects.

Baclofen, for example, is an antispasticity drug that contains both GABA and phenylethylamine moieties. ${ }^{7,22}$ Awaad studied baclofen $(n=264)$ or botulinum toxin type A $(\mathrm{n}=186)$ in patients age $\leq 18$ years with tics or TS $\left(n=450\right.$ total). ${ }^{7}$ Treatment with baclofen or botulinum toxin type A was effective, safe, and reliable. In responsive patients, the therapy produced dramatic results as determined by standard assessments of tics. The most common side effects in the group treated with baclofen were sedation and drowsiness. Soreness and mild muscle weakness were the most commonly reported adverse events in the group treated with botulinum toxin A. A separate study, which evaluated baclofen in a small number of patients $(\mathrm{n}=10)$ with tics, produced similar results. ${ }^{22}$

The atypical GABAergic effects of levetiracetam provide the rationale for treatment of patients with tics. ${ }^{11,12,23}$ By inhibition of zinc and beta carbolines in a manner that interrupts chloride influx, levetiracetam may enhance chloride ion influx at the GABA-A receptor complex.

In this open-label study, the effect of levetiracetam on motor and vocal tics was evaluated in a population of 60 pediatric patients with tics and TS. In addition, the effects of levetiracetam therapy on measures of behavior and school performance were recorded. The results demonstrate that treatment with levetiracetam improved clinical outcomes in all 60 patients. A total of 43 patients showed improvement in their behavior and school performance. It must be noted that the interpretation of these results is limited by the open-label study design. The variability in symptoms of TS must also be considered when interpreting results of clinical studies in these patients.

Levetiracetam may be a well-tolerated and relatively safe alternative to currently used therapies..$^{13-15}$ The most commonly reported side effects associated with use of levetiracetam are central nervous system effects, including somnolence and fatigue. Reports of the use of levetiracetam in children are limited, ${ }^{13,16-19,24}$ but behavioral side effects have been reported. ${ }^{25,26}$ In some patients, behavioral effects resolved with reductions in dosages or discontinuation of therapy. Patients being treated with levetiracetam should be monitored for such side effects, and care should be taken with dose titration. Another pharmacologic property of levetiracetam is the absence of hepatic metabolism, which is associated with a very low potential for drug interactions. ${ }^{14}$ This finding is an important factor for patients who have coexisting conditions, such as $\mathrm{ADHD}$, that require use of an additional medication.

Whenever drug therapy is used in children with tics, it is important to monitor safety and tolerability as well as efficacy. In addition, patients, family members, and school personnel should be educated about the characteristics of TS. Patients and their families can also benefit from counseling and participation in support groups.

These preliminary results suggest that levetiracetam may be useful in treating tics in children and adolescents. Levetiracetam may be a candidate for additional evaluations in patients who have both TS and epilepsy. The established safety profile of levetiracetam makes this drug an attractive alternative to other agents, which are associated with the potential for serious side effects. In addition, the low risk of drug interactions with levetiracetam might be a consideration for patients who require the concurrent use of other medications to treat comorbid conditions, such as ADHD.

Despite the limitations of the open-label study design, the results presented here provide a rationale for initiation of larger, double-blind, controlled studies of levetiracetam in children and adolescents. Although drugs are studied in adults according to the guidelines established by regulatory agencies, few drugs, including those to treat tics and TS, have been adequately evaluated in pediatric populations. In addition, other trials to more fully elucidate the pathophysiology of TS could help lead to targeted drug development and optimal drug therapy.

\section{REFERENCES}

1. Scahill L, Tanner C, Dure L. The epidemiology of tics and Tourette syndrome in children and adolescents. Adv Neurol 2001; 85:261-271.

2. Pringsheim T, Davenport WJ, Lang A. Tics. Curr Opin Neurol 2003;16:523-527.

3. Castiglia PT. Tourette syndrome. J Pediatr Health Care 1997;11: 189-191.

4. Spencer T, Biederman J, Coffey B, Geller D, Faraone S, Wilens T. Tourette disorder and ADHD. Adv Neurol 2001;85:57-77.

5. Sandor P. Pharmacological management of tics in patients with TS. J Psychosom Res 2003;55:41-48.

6. Lavenstein BL. Treatment approaches for children with Tourette syndrome. Curr Neurol Neurosci Rep 2003;3:143-148.

7. Awaad Y. Tics in Tourette syndrome: new treatment options. J Child Neurol 1999;14:316-319.

8. Silva RR, Munoz DM, Daniel W, Barickman J, Friedhoff AJ. Causes of haloperidol discontinuation in patients with Tourette's disorder: management and alternatives. J Clin Psychiatry 1996;57: $129-135$.

9. Mink JW. Basal ganglia dysfunction in Tourette syndrome: a new hypothesis. Pediatr Neurol 2001;25:190-198.

10. Goetz CG. Clonidine and clonazepam in Tourette syndrome. Adv Neurol 1992;58:245-251. 
11. Poulain P, Margineanu DG. Levetiracetam opposes the action of GABAA antagonists in hypothalamic neurones. Neuropharmacology 2002;42:346-352.

12. Rigo JM, Hans G, Nguyen L, et al. The antiepileptic drug levetiracetam reverses the inhibition by negative allosteric modulators of neuronal GABA- and glycine-gated currents. Br J Pharmacol 2002; 136:659-672.

13. French J. Use of levetiracetam in special populations. Epilepsia 2001;42(Suppl. 4):40-43.

14. Patsalos PN. Pharmacokinetic profile of levetiracetam: toward ideal characteristics. Pharmacol Ther 2000;85:77-85.

15. Abou-Khalil B, Lazenby B. Long-term experience with levetiracetam. Epileptic Disord 2003;5(Suppl. 1):S33-S37.

16. Glauser TA, Dulac O. Preliminary efficacy of levetiracetam in children. Epileptic Disord 2003;5(Suppl. 1):S45-S50.

17. Miller SG. Efficacy and safety of levetiracetam in pediatric migraine. Headache 2004;44:238-243.

18. Herranz JL, Rufo-Campos M, Arteaga R. Effectiveness and tolerability of levetiracetam in 43 children and adolescents with epilepsy [in Spanish]. Rev Neurol 2003;37:1005-1008.

19. Lagae L, Buyse G, Deconinck A, Ceulemans B. Effect of levetiracetam in refractory childhood epilepsy syndromes. Eur J Paediatr Neurol 2003;7:123-128.
20. Diagnostic and Statistical Manual of Mental Disorders DSM-IVTR. 4th ed. Arlington, VA: American Psychiatric Association; 2000.

21. McConville BJ, Sorter MT. Treatment challenges and safety considerations for antipsychotic use in children and adolescents with psychoses. J Clin Psychiatry 2004;65(Suppl. 6):20-29.

22. Singer HS, Wendlandt J, Krieger M, Giuliano J. Baclofen treatment in Tourette syndrome: a double-blind, placebo-controlled, crossover trial. Neurology 2001;56:599-604.

23. Margineanu DG, Klitgaard H. Levetiracetam has no significant gamma-aminobutyric acid-related effect on paired-pulse interaction in the dentate gyrus of rats. Eur J Pharmacol 2003;466:255261.

24. Frost MD, Gustafson MC, Ritter FJ. Use of levetiracetam (lev) in children under two years of age [abstract]. Epilepsia 2002; 43(Suppl. 7):57.

25. White JR, Walczak TS, Leppik IE, et al. Discontinuation of levetiracetam because of behavioral side effects: a case-control study. Neurology 2003;61:1218-1221.

26. Keppra (levetiracetam) prescribing information. Smyrna, GA: UCB Pharma; 2004. 